\title{
Treatment of latent $M$. tuberculosis infection and use of antiretroviral therapy to prevent tuberculosis
}

\author{
Timothy R. Sterling ${ }^{1}$ and Philana Ling Lin ${ }^{2}$ \\ 'Vanderbilt University Medical Center, Division of Infectious Diseases, Department of Medicine, Nashville, Tennessee, USA. Department of Pediatrics, University of Pittsburgh, Pittsburgh, \\ Pennsylvania, USA.
}

\begin{abstract}
Even with treatment of HIV with antiretroviral therapy (ART), the risk of tuberculosis (TB) reactivation remains higher in HIV-infected than HIV-uninfected persons. In this issue of the JCI, Ganatra et al. explored TB reactivation in the context of ART using TB and simian immunodeficiency virus-coinfected (TB/SIV-coinfected) nonhuman primates. The authors found that treating rhesus macaques with ART restored $C D 4^{+} \mathrm{T}$ cells in whole blood, spleen, and bronchoalveolar lavage (BAL) fluid, but not in the lung interstitium. TB risk was not decreased in the coinfected macaques treated with ART for 14-63 days, suggesting that ART does not decrease the short-term risk of reactivation. Reactivation occurred as CD4 $T$ cells were increasing, which is consistent with observations made in humans. This study provides a potential model for systematic evaluation of TB/SIV coinfection and different treatment regimens and strategies to prevent TB reactivation.
\end{abstract}

\section{Latent Mycobacterium tuberculosis infection}

To facilitate the treatment and prevention of tuberculosis (TB), it is important to fully understand TB pathogenesis. Latent Mycobacterium tuberculosis infection is the most common clinical manifestation of this pathogen, in which infected persons are asymptomatic. However, there is a spectrum of clinical manifestations (1). To prevent $\mathrm{TB}$, it is of particular importance to understand the transition from latent M. tuberculosis infection to subclinical and symptomatic TB disease, so that preventive therapy can be developed, evaluated, appropriately targeted, and optimized. In humans, HIV infection is the strongest risk factor for progression to active $\mathrm{TB}$ among persons with $M$. tuberculosis infection (2). Both antiretroviral therapy (ART) to treat HIV and restore immune function, and isoniazid preventive therapy to treat
M. tuberculosis infection, independently decrease the risk of progressing from $M$. tuberculosis infection to TB disease (refs. 3, 4 and Figure 1).

The initial and subsequently standard treatment of latent $M$. tuberculosis infection has been six to nine months of isoniazid. More recently, shorter rifamycinbased treatment regimens have been preferred because of their safety and efficacy, higher treatment completion rates, and therefore likely increased effectiveness compared with six to nine months of isoniazid $(5,6)$. A previous study from the Rengarajan and Kaushal groups showed that three months of weekly isoniazid plus rifapentine effectively cleared $M$. tuberculosis infection in macaques, providing evidence of the efficacy of this regimen (7), consistent with the efficacy and effectiveness demonstrated in human clinical trials $(8,9)$. Treatment regimens even

Related Article: p. 5171

Conflict of interest: The authors have declared that no conflict of interest exists.

Copyright: (c) 2020, American Society for Clinical Investigation.

Reference information: J Clin Invest. 2020;130(10):5102-5104. https://doi.org/10.1172/JCI137294.

shorter than three to four months have been evaluated recently, including one month of daily isoniazid plus rifapentine (10). A six-week regimen of daily rifapentine is currently under investigation (NCT03474029; https://clinicaltrials. gov/ct2/show/NCT03474029). However, several questions remain regarding the treatment of latent $M$. tuberculosis infection. Future studies should address the optimal treatment duration, total rifamycin exposure, and treatment intermittency. Therefore, an animal model that accurately predicts treatment response in humans would be highly valuable. Data from a murine model predicted that the treatment efficacy in humans corresponded to six to nine months of isoniazid, three to four months of rifampin, three months of weekly isoniazid plus rifapentine, and two months of rifampin plus pyrazinamide (11-16). However, the mouse model has limitations, including lack of a latent state and lack of the granuloma architecture and types seen in humans (17).

\section{Preventing reactivation of}

latent $M$. tuberculosis infection In this issue of JCI, Ganatra et al. (18) assessed whether ART alone and the associated immune reconstitution would prevent reactivation of latent $M$. tuberculosis infection. Macaques were first infected with $M$. tuberculosis for nine weeks and then infected with simian immunodeficiency virus (SIV); a subset of the latter group received ART four weeks after SIV infection. The duration of ART ranged from 14-63 days in the four macaques that received it, and all macaques were followed for up to 24 weeks. ART was associated with a reduction in SIV viral load and increased $\mathrm{CD} 4{ }^{+} \mathrm{T}$ cell counts in the blood, but ART-treated macaques failed to decrease the risk of $\mathrm{TB}$ reactivation compared with the ART-naive group; the relative risk (RR) of TB actually increased, though it was not statistically significant 


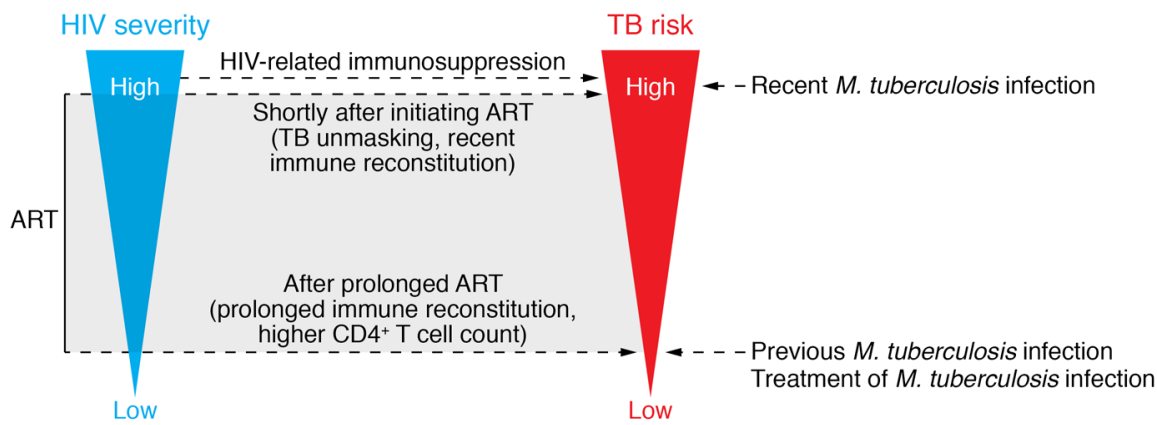

Figure 1. Factors associated with $M$. tuberculosis and HIV coinfection that affect the risk of developing active TB. In TB/HIV-coinfected individuals, recent infection with $M$. tuberculosis, longer duration of HIV infection, associated lower CD4+ $T$ cell counts, and recent HIV ART increase the risk for developing TB. Conversely, treatment for $M$. tuberculosis infection, infection with $M$. tuberculosis before subsequent reexposure to TB, and prolonged HIV ART decrease the risk for TB. Notably, immune recovery relates to the risk for reactivation; restoring effective resident $T$ cells in the lungs may require longer ART durations. These factors could be evaluated in the macaque model used in Ganatra et al. (18) to assess ways to optimally decrease TB risk.

$(\mathrm{RR}=1.23$; 95\% CI 0.97-1.5). The mycobacterial burden in the lungs and spleen was similar in the two groups. Notably, $\mathrm{CD} 4^{+} \mathrm{T}$ cell counts in the lungs remained low in the macaques that received ART, highlighting the importance of tissue-resident immune responses and their possible discordance with systemic responses in the blood. This is particularly relevant to $\mathrm{TB}$, as blood responses are not a reliable marker of immune responses within the granuloma (19). These findings are consistent with data from humans indicating that ART alone is insufficient to prevent progression to TB among individuals with $M$. tuberculosis infection. The Ganatra et al. data (18) are also consistent with the finding that isoniazid preventive therapy provides an additional protective effect to ART $(3,4)$. In most humans with $M$. tuberculosis and HIV infection, it is unclear which infection occurred first. Thus, translational models like this allow the field to directly address questions that would otherwise be impractical or unethical to research in human populations and allow us to evaluate additional questions regarding $M$. tuberculosis pathogenesis and treatment.

\section{Conclusions and remaining questions}

No model is perfect and there are important limitations to consider when deciding whether the Ganatra et al. (18) results in macaques can be extended to humans. In most cases of asymptomatic or latent infection in humans, it is assumed that
M. tuberculosis infection has been established for months to years. Nine weeks of infection in a macaque may not reflect the same immune response as that seen in a human with latent $M$. tuberculosis infection for several years before HIV infection. Reactivation of TB among all four ART-treated macaques with SIV occurred after only 14-63 days of ART. Given the short period of ART, it is possible that such treatment resulted in activation of already ongoing subclinical TB (18). Symptoms of active $\mathrm{TB}$ during immune reconstitution with ART has been described in humans - the so-called "unmasking of TB" $(20$, 21). Indeed, ART-treated animals showed some evidence of $\mathrm{CD} 4^{+} \mathrm{T}$ cell count recovery in the blood and bronchus-associated lymphoid, albeit there were only four animals in this group (18). These results suggest that ART did not prevent short-term reactivation risk. However, the long-term implications remain unknown; restoring lung-resident $\mathrm{T}$ cells that are critical to containing M. tuberculosis infection and preventing dissemination may require longer ART durations (Figure 1). Studies that vary ART duration may provide insight into the balance of pro- and antiinflammatory responses that play a critical role in both HIV and TB immune pathology.

The study by Ganatra et al. (18) raises several important questions: What does "immune reconstitution" really mean? While this term is often used when systemic viral load is dramatically reduced and $\mathrm{CD}^{+} \mathrm{T}$ cell counts start to increase, recovery of pathogen-specific immune responses is usually not assessed; the latter would have been a valuable metric in this study. A larger sample size would give greater confidence in the results and also allow for the identification of intermediate differences in immune responses. The results from Ganatra et al. (18) suggest that one important factor associated with $\mathrm{TB}$ reactivation was the persistently low $\mathrm{CD} 4^{+}$ $\mathrm{T}$ cell count in the lungs, yet reactivation is not solely dependent on $\mathrm{CD} 4^{+} \mathrm{T}$ cell counts $(22,23)$. Lower $\mathrm{CD} 4^{+} \mathrm{T}$ cell counts in the lung are consistent with the observation that even persons with well-controlled HIV infection (>700 $\mathrm{CD}^{+}$lymphocytes/ $\mathrm{mm}^{3}$ ) have a TB risk that exceeds that seen in HIV-seronegative persons (24). Given that current ART is highly effective, safe, and increasingly available globally, understanding host immune responses to M. tuberculosis after prolonged ART and the resultant immune restoration and the timing of such restoration are important. Characterization of any persistent defects in the immune response to M. tuberculosis - or the lack of such defects - could inform TB prevention strategies.

Does the relative timing of HIV and $M$. tuberculosis infection affect TB risk? Similarly, what if there were more advanced immunosuppression before initiation of ART, rather than starting ART shortly after SIV infection? The median peripheral blood $\mathrm{CD}^{+} \mathrm{T}$ cell percentage in the macaques studied by Ganatra et al. (18) was high (suggesting good immune function), even in those with SIV that were not treated with ART $\left(\mathrm{CD} 4^{+} \mathrm{T}\right.$ cell percentage $>40)$. As the authors point out, reactivation still occurred despite such a short period of SIV infection (18). Fluctuations in immune dysregulation and their impact on the immune response to M. tuberculosis may differ substantially early after SIV infection compared with long-term SIV infection, as may be the case in human HIV infection. Such a controlled experimental model can directly address these questions and facilitate the identification of biomarkers associated with TB risk and optimized treatment.

Can we use these models to better understand latent M. tuberculosis infection, its treatment, and the complex interactions with HIV infection? And how well will the results reflect human TB/HIV 
coinfection? Although it is not financially feasible to maintain nonhuman primates for years after M. tuberculosis infection, the duration of infection probably plays a key role in the spectrum of latency and risk of TB reactivation. That said, other nonhuman primate models of TB have shown that a spectrum of latent infection exists, and this spectrum has important implications for reactivation risk (25). Previous human studies indicate that latent M. tuberculosis infection is associated with a reduced risk of progression to active $\mathrm{TB}$ upon subsequent reexposure to $M$. tuberculosis (26). This association is consistent with a recent nonhuman primate study showing that ongoing primary $M$. tuberculosis infection could protect against reinfection with the same strain (27). It remains unclear how long this protective effect against subsequent infection (in both immunocompetent and immunocompromised individuals) might last after remote latent $M$. tuberculosis infection, and whether the protection is lost after treatment of latent $M$. tuberculosis infection. In other words, does the treatment of latent M. tuberculosis infection in immune-competent hosts actually increase the risk of active TB in persons who are subsequently reexposed to $M$. tuberculosis? For those with HIV infection, does the risk of TB reactivation without treatment for latent infection (but with ART) outweigh the potential benefit? Answers to the above questions would help identify the most effective preventive strategies and thereby facilitate TB elimination.

Address correspondence to: Timothy R. Sterling, Vanderbilt University Medical Center, Division of Infectious Diseases, Department of Medicine, A2209 Medical Center North, 1161 21st Avenue South, Nashville, Tennessee 37232, USA. Phone: 615.322.2035; Email: timothy.sterling@ vumc.org

1. Esmail H, Barry CE, Young DB, Wilkinson RJ. The ongoing challenge of latent tuber- culosis. Philos Trans R Soc Lond, B, Biol Sci. 2014;369(1645):20130437.

2. WHO Global Tuberculosis Program. WHO consolidated guidelines on tuberculosis: module 1 : prevention: tuberculosis preventive treatment. https://www.who.int/publications/i/item/ who-consolidated-guidelines-on-tuberculosismodule-1-prevention-tuberculosis-preventivetreatment. Updated March 24, 2020. Accessed July 29, 2020.

3. Rangaka MX, et al. Isoniazid plus antiretroviral therapy to prevent tuberculosis: a randomised double-blind, placebo-controlled trial. Lancet. 2014;384(9944):682-690.

4. TEMPRANO ANRS 12136 Study Group, et al. A trial of early antiretrovirals and isoniazid preventive therapy in Africa. N Engl J Med. 2015;373(9):808-822.

5. Sterling TR, et al. Guidelines for the treatment of latent tuberculosis infection: recommendations from the National Tuberculosis Controllers Association and CDC, 2020. MMWR Recomm Rep. 2020;69(1):1-11.

6. Horsburgh CR, et al. Latent TB infection treatment acceptance and completion in the United States and Canada. Chest. 2010;137(2):401-409.

7. Foreman TW, et al. Isoniazid and rifapentine treatment eradicates persistent Mycobacterium tuberculosis in macaques. Am J Respir Crit Care Med. 2020;201(4):469-477.

8. Martinson NA, et al. New regimens to prevent tuberculosis in adults with HIV infection. N Engl JMed. 2011;365(1):11-20.

9. Sterling TR, et al. Three months of rifapentine and isoniazid for latent tuberculosis infection. NEngl JMed. 2011;365(23):2155-2166.

10. Swindells S, et al. One month of rifapentine plus isoniazid to prevent HIV-related tuberculosis. N Engl JMed. 2019;380(11):1001-1011.

11. Grosset J, Lounis N, Truffot-Pernot C, O'Brien RJ, Raviglione MC, Ji B. Once-weekly rifapentine-containing regimens for treatment of tuberculosis in mice. Am J Respir Crit Care Med. 1998;157(5 Pt 1):1436-1440.

12. Gordin F, et al. Rifampin and pyrazinamide vs isoniazid for prevention of tuberculosis in HIV-infected persons: an international randomized trial. Terry Beirn Community Programs for Clinical Research on AIDS, the Adult AIDS Clinical Trials Group, the Pan American Health Organization, and the Centers for Disease Control and Prevention Study Group. JAMA. 2000;283(11):1445-1450.

13. Schechter M, et al. Weekly rifapentine/isoniazid or daily rifampin/pyrazinamide for latent tuberculosis in household contacts. Am J Respir Crit Care Med. 2006;173(8):922-926.

14. Zhang T, Zhang M, Rosenthal IM, Grosset JH, Nuermberger EL. Short-course therapy with daily rifapentine in a murine model of latent tuberculosis infection. Am J Respir Crit Care Med. 2009;180(11):1151-1157.

15. Zhang T, Li SY, Williams KN, Andries K, Nuermberger EL. Short-course chemotherapy with TMC207 and rifapentine in a murine model of latent tuberculosis infection. Am J Respir Crit Care Med. 2011;184(6):732-737.

16. Menzies D, et al. Four months of rifampin or nine months of isoniazid for latent tuberculosis in adults. N Engl J Med. 2018;379(5):440-453.

17. Shi C, Shi J, Xu Z. A review of murine models of latent tuberculosis infection. Scand J Infect Dis. 2011;43(11-12):848-856.

18. Ganatra, et al. Antiretroviral therapy does not reduce tuberculosis reactivation in a tuberculosis-HIV coinfection model. J Clin Invest. 2020;130(10):5171-5179.

19. Gideon HP, et al. Variability in tuberculosis granuloma T cell responses exists, but a balance of pro- and anti-inflammatory cytokines is associated with sterilization. PLoS Pathog. 2015;11(1):e1004603.

20. Meintjes G, et al. Tuberculosis-associated immune reconstitution inflammatory syndrome: case definitions for use in resource-limited settings. Lancet Infect Dis. 2008;8(8):516-523.

21. Lawn SD, Wilkinson RJ, Lipman MC, Wood R. Immune reconstitution and "unmasking" of tuberculosis during antiretroviral therapy. Am J Respir Crit Care Med. 2008;177(7):680-685.

22. Lin PL, et al. CD4 T cell depletion exacerbates acute Mycobacterium tuberculosis while reactivation of latent infection is dependent on severity of tissue depletion in cynomolgus macaques. AIDS Res Hum Retroviruses. 2012;28(12):1693-1702.

23. Foreman TW, et al. CD $4^{+}$T-cell-independent mechanisms suppress reactivation of latent tuberculosis in a macaque model of HIV coinfection. Proc Natl Acad Sci USA. 2016;113(38):E5636-E5644.

24. Gupta A, Wood R, Kaplan R, Bekker LG, Lawn $\mathrm{SD}$. Tuberculosis incidence rates during 8 years of follow-up of an antiretroviral treatment cohort in South Africa: comparison with rates in the community. PLOS ONE. 2012;7(3):e34156.

25. Lin PL, et al. PET CT identifies reactivation risk in cynomolgus macaques with latent $M$. tuberculosis. PLoS Pathog. 2016;12(7):e1005739.

26. Andrews JR, Noubary F, Walensky RP, Cerda R, Losina E, Horsburgh CR. Risk of progression to active tuberculosis following reinfection with Mycobacterium tuberculosis. Clin Infect Dis. 2012;54(6):784-791.

27. Cadena AM, et al. Concurrent infection with Mycobacterium tuberculosis confers robust protection against secondary infection in macaques. PLoS Pathog. 2018;14(10):e1007305. 